\title{
1 Multiscale light-sheet organoid imaging framework
}

2

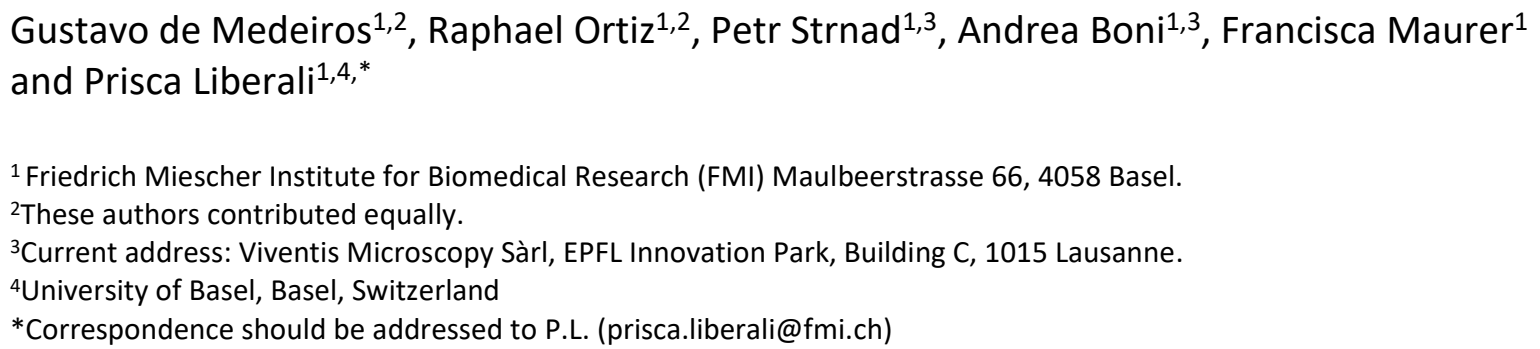

\section{Abstract}

We present an imaging framework capable of turning long term light-sheet imaging of organoids into digital organoids. The framework takes advantage of deep learning techniques to faithfully segment single organoids, their lumen, cells and nuclei in 3D and over long periods of time. In parallel, large lineage trees for each organoid are predicted and corrected to iteratively improve the tracking and segmentation performances over time. To visualize the extracted information, we developed a web-based "Digital Organoid Viewer" that allows a unique understanding of the multivariate and multiscale data by linking $2 \mathrm{D}$ lineage trees with the corresponding 3D segmentation meshes.

We also backtracked single cells of interest after fixation obtaining detailed information about their history within the entire organoid context. Furthermore, we show nuclei merging events that arise from cytokinesis failure and that these polyploid never reside in the intestinal crypt, hinting at a tissue scale control and feedback on cellular fidelity. Molecularly, these cytokinesis failures depend on a regenerative state of the organoids and are regulated by Lats 1 and RXR and we propose a model of tissue integrity by multi-scale check points. This discovery sheds light on the robustness of a regenerative YAP cellular state, questioning the role of polyploidy in intestinal regeneration.

\section{Main}

During adult life, organs such as the intestine are challenged to diverse environmental conditions, requiring the tissue to be robust and yet plastic. For instance, during regeneration after damage, surviving cells need to carefully orchestrate a fast and robust regrowth process in coordination with proper shape recovery as well as functional and morphological remodeling. To best overcome the difficulties surrounding the study of these tissue dynamics in inner organs in vivo, organoids have become a powerful experimental method owing to their exceptional accessibility and manipulability ${ }^{1-3}$. For the case of the intestinal tract, intestinal organoids grown from single cells functionally recapitulate both the regenerative response of the intestinal epithelium as well as the homeostasis of the in vivo intestine ${ }^{4}$. 
Morphologically, they also recapitulate the main dynamics of crypt formation, making them a unique in vitro system ${ }^{5,6,7,8}$.

Although with high degree of accessibility, performing live imaging of organoid growth remains a challenge, as it typically requires not only microscopy techniques capable of stable long-term imaging of several samples simultaneously, but also dedicated analysis and processing pipelines that can cope with complex imaging data. Nonetheless, previous work on the live recording of organoid dynamics has focused either on specific biological questions $s^{6,8,9}$ or on specific isolated tools ${ }^{10}$ without a more generalised yet in-depth approach on light-sheet imaging and data analysis. The only current work which aimed at creating a light-sheet organoid imaging platform ${ }^{11}$ focused mainly on the determination of culture-wide heterogeneities through a combination of both light-sheet and wide-field techniques. Although showing organoid diversity within the same culture, in-depth cellular multi-scale analysis for each organoid remained lacking.

To fully bridge the gap and provide quantitative information on organoid growth dynamics with in-depth cellular analysis, we here provide a unified multiscale light-sheet imaging framework tailored to live organoid imaging called LSTree. Our framework encompasses preprocessing, automated lineage tracking, segmentation and multivariate feature extraction pipelines which provide multiscale measurements from organoid to single cell levels. By focusing on the development of intestinal organoids, we show that this holistic set of tools allows the combination of whole organoid and single cell features to be analysed simultaneously, having both lineage tree as well as spatial segmentation information presented in a clear and unified way. We demonstrate that our pipeline is compatible with fixation and immunolabeling after live imaging by tracking back cells positive for specific markers and compare the history of these cells with all other cells in the organoid. Further, we use our framework to dissect new biological insights on the role of polyploidy during intestinal organoid growth, and we propose a tissue level check point for tissue integrity that could start explaining the interplay between regeneration and cancer.

\section{Results}

\section{Imaging framework for light-sheet microscopy of organoid growth}

We developed a multiscale imaging framework that comprehends acquisition, preprocessing, automated tracking, segmentation with further feature extraction as well as visualization dedicated for 3D live imaging. In this work, we applied our framework to live intestinal organoid light-sheet recordings performed with a dual-illumination inverted lightsheet ${ }^{6}$ microscope, which utilizes a multi-positioning sample holder system (Fig. 1a). In order to image organoid development, we followed previously published protocols ${ }^{6}$, FACS sorting single cells from mature organoids and mounting them as $5 \mathrm{uL}$ mix drops with Matrigel on top of a ca. 50 um thick fluorinated ethylene propylene (FEP) foil, which are then covered in medium (Fig 1b left and Methods Section). We patterned the FEP foil used for mounting in order to create small wells (Supplementary Figure 1), allowing better control of the sample position within the holder, while improving reproducibility of experiments by preventing drops from being washed away during medium change of fixation procedures. As previously demonstrated, the microscope we utilized in all demonstrations is capable of imaging live 
intestinal organoids for long periods of time $e^{6,8}$, as well as acquiring time-lapses of mouse embryonic and gastruloid development ${ }^{12,13}$. However, one important drawback of the system was that the alignment of the illumination beams is done only once, prior to the experiment and irrespective of the position of the sample in the dish or holder. Although sufficient in certain situations (e.g. mouse embryo imaging), imaging of samples embedded and distributed inside a gel suffer from refractive index mismatch between water and Matrigel, as well as from the presence of other obstacles in the light-path (other organoids or debris) and from the curved shape of the sample holder itself. Therefore, to improve recording conditions in every individual sample, we developed a position dependent illumination alignment step. This allows to fine tune the alignment of each of the illumination sheets in respect to the detection plane for every sample position so that best image quality possible can be achieved throughout (Fig $\mathbf{1 b}$ right). Moreover, to minimize storage needs, acquired images were compressed on the fly (using standard LZW library), and the saved data were then further cropped (Fig 1c upper row). To better address low signal-to-noise (SNR) recordings we also added denoising and deconvolution steps. Denoising is done using the Noise2Void scheme ${ }^{14}$, with its output sent to a tensor-flow based image deconvolution ${ }^{15}$ (https://github.com/hammerlab/flowdec) (Fig 1c lower row) using measured PSFs from beads (Supplementary Note 1 and Methods Section).

With these first modules at hand, we imaged organoids expressing Histone 2B and mem9 membrane peptide tagged with mCherry and GFP respectively, recording the growth and development of several organoids for around 4 days starting from single cells up to 4-cell clusters (Fig 1d and Supplementary Movie $\mathbf{1}$ ) every 10 minutes. The collected data comprised of organoids that form both budding and enterocyst phenotypes: whereas budding organoids grow from single cells into mature organoids with both crypt and villus structures, enterocysts, comprised of terminally differentiated enterocytes, do not have crypts as they do not develop Paneth cells required for the establishment of the stem cell niche, a necessary step for crypt formation ${ }^{6,8}$.

To extract 3D information of the organoid dynamics throughout growth, we performed partial semi-automatic tracking using the Fiji plugin Mastodon (https://github.com/mastodon$\mathrm{sc} /$ mastodon) on 7 datasets. After that, we extracted initial features based on organoid and single cell segmentation and plotted this data over time, to get a first overview on how these parameters evolved (Fig 1e). For example, we noticed large variability in cell division synchronicity, as in some datasets the nuclei number growth over time loses the typical staircase-like behavior already early on. Although epithelium volume growth curves follow that of nuclei number, with the characteristic exponential behavior, nuclei density slightly increases over time. Mean cell volume showed characteristic mitotic peaks, with overall cell volume decrease over time, matching the increase in nuclei density. Interestingly, although initially cell to nuclei volume ratio vary, all datasets converge to common steady state values where the cell volume is ca. 3 to 4 times larger than the nuclear volume. We also observed a consistent change in organoid volume due to medium change during the live recordings (Supplementary Figure 2 and Supplementary Note 3). As this initial assessment of our imaging data showed consistent and reproducible results, and to be able to handle larger dataset more rapidly, we developed a more integrated and automated approach. 
128 To make the tools directly accessible, we decided to incorporate all image processing and 129 data analysis modules into a unified framework, named LSTree (https://github.com/fmi130 basel/LSTree) and is implemented as a Luigi (https://github.com/spotify/luigi) workflow. As pre-processing first step, the user selects which organoid needs to be cropped on the last frame of a movie and minimal bounding boxes per time-point as well as global bounding boxes are automatically generated for all time-points (Fig 2a). We also implemented an interactive tool to review the crops and perform few manual corrections, e.g. to account for large displacement between consecutive frames due to a medium change (Supplementary Figure 3). Next, denoising and deconvolution of cropped and registered movies is performed as one combined step.

For the segmentation of organoids, as well as their cells and nuclei, we adopted different segmentation strategies all relying on convolutional neural networks (Fig $\mathbf{2 b}$ ) and utilizing the RDCNet instance segmentation network as a base ${ }^{16}$. Nuclei are segmented in 3D following a deep learning model trained with a mix of complete and partial annotations. A small subset of the frames is fully annotated by manually expanding the labels to the full nuclei, whereas partial annotations rely on the initial tracking performed with Mastodon by drawing spheres at the position of tracked nuclei. (Fig $\mathbf{2 b}$ upper row). Jupyter notebooks for interactive visualization and correction of the predicted segmentation are also provided, which allows improving the model accuracy with minimal annotating time.

Organoid and cell segmentation also use RDCNet and leverages the pre-computed nuclei segmentation to avoid manual annotations of individual cells. The semantic branch predicts 3 classes (background, lumen and epithelium) and is supervised by manual annotations of a few frames per datasets. To subdivide the epithelium mask into cells, the previously segmented nuclei are used as partial cell annotations under the assumption that they are randomly distributed within the cell compartment (Fig $\mathbf{2 b}$ lower row, Supplementary Note 2). Finally, in addition to the segmentation volumes and nuclei number (Fig. 1e), several different features is also extracted such as nuclear distance to apical/basal membranes, fluorescence intensity, distance to parent node, number of neighbors per cell and more (For a complete list of features with short explanations see Supplementary Table 1).

\section{Deep learning model for automated lineage tracing}

Although suitable for estimating lineage trees for a few datasets, semi-automated tracking of many large datasets with the Mastodon Fiji plugin can be extremely time consuming. To significantly improve this process, we trained and refined a deep learning model on the available tracked datasets to automatically generate candidate trees that only require minimal corrections (Fig 2c,d, Supplementary Note 2). The most effective lineage tracing algorithms to date attempt to first detect nuclei and subsequently link them across time while enforcing a complex set of rules ${ }^{17-19}$. We however found that this approach performed poorly on later time-points where the detection accuracy dropped due to the high cell density. We realized that on these challenging cases, even manual nuclei detection requires to take in account the temporal information from neighboring frames. Therefore, we developed a joint segmentation-tracking approach that simultaneously predicts matching nuclei labels on 2 consecutive frames. To this end, we extended the RDCNet instance segmentation model to predict pseudo 4D labels (3D convolutional network with time axis as an additional image 
172 channels) mapping correspondences between nuclei in 2 consecutive frames (Fig 2c).

173 Predicting linked nuclei segmentations has the advantage to enforce constancy over the

174 entire nuclear volume rather than relying on an ambiguous center, as well as implicitly

175 enforcing rules such as minimum cell distance or plausible nuclei volume constraints in a data-

176 driven manner. This is a unique method as it keeps the number of manual hyper-parameters

177 tuning to a minimum and can be improved over time as more validated and corrected

178 datasets are incorporated in the training set.

179 To assemble the predicted tree, nuclei labels in each frame are connected to their parents in 180 the previous frame by finding the linking label with the maximum overlap (Fig 2c, 181 Supplementary Figure 4). The predicted tree is then saved with the structure of a MaMuT 182 .xml track file, which can be imported into Mastodon for further correction (Fig 2d). As a direct consequence of the joint segmentation, additional information such as the nuclei volume can be overlaid on the predicted trees to aid in the curation process (Supplementary Figure 4, Supplementary Note 2). For instance, jumps in nuclear volume highlight positions where tracks should be merged or split. The manual curation time ranges from minutes to a couple hours on the most challenging datasets (e.g. low SNR images, abnormal nuclei shape). In summary, this novel and efficient tree prediction approach allows high quality prediction of organoid lineage trees with long tracks spanning multiple division cycles (up to 5 generations in this work) enabling tracked data to cross spatiotemporal scales.

\section{Digital organoid viewer}

With the lineage trees and the deep learning 4D segmentation of organoid, lumen, cells and nuclei at hand, we created a multiscale digital organoid viewer to explore and perform indepth data mining. The viewer combines both lineage trees and segmented meshes, facilitating the direct comparison of different features within a multiscale digital organoid framework. We also added the possibility to overlay recorded images with the meshes allowing a direct inspection of the predicted segmentation (Fig 3a, Supplementary Movie 2). When hovering a node with the mouse, its associated features are displayed, selected nodes are interactively highlighted on the meshes, and color coding of both trees and meshes can be assigned independently, so that same or complementary features can be visualized at once.

203 As an example of the analysis and visualization tools presented above, nuclear volume quantifications can be evaluated directly onto the tree of a specific dataset (Fig $\mathbf{3 b}$ ). Using this approach, it is possible to observe and quantify how much nuclei volumes change with each generation and over time, with the smallest volumes observed right after division. Similarly, we can observe that the nuclear distance to the basal membrane (Fig 3c) increases due to interkinetic nuclear migration towards the apical side. Combining the same visualization procedure with the segmented meshes, we can render the nuclei or cells in 3D, using the same color-coding as for feature on the trees (Fig 3d). Last but not least, we can also compare the extracted features against the general trend from all other datasets combined, allowing us a direct evaluation of variability across experiments (Fig 3e,f). This way we can for example evaluate the increased distancing of nuclei from the apical membrane, a known effect due to epithelial polarization (Fig 3f).

215 In summary, this is a unique set of tools which allows not only multiscale segmentation of organoids along with lineage tree predictions, but also the simultaneous visualization of both 
217 trees and segmented meshes into a unified web-viewer. All steps of the process are

218 implemented to keep storage, memory, and manual tuning requirements to a minimum, making this a powerful and yet lightweight light-sheet based framework.

\section{Functional imaging through fixation and backtracking}

222 Next, we analyzed functional information on the tracked cells and organoids contained in the lineage trees. Although our imaging framework allows the visualization and quantification of a large number of features at the cellular and organoid levels throughout organoid growth, functional information remains dependent on the currently existing fluorescent organoid lines. The easiest way to theoretically approach this is to perform stable multicolor live imaging for long periods of time. However, overlapping emission/excitation spectra limits the total number of fluorescent reporters and concerns regarding interference with the normal cell function, signal-to-noise sensitivity for low abundant proteins, photostability and general phototoxicity due to laser illumination limit the use of fluorescent reporters. To overcome this, we integrated fixation and immunolabelling steps after the live recordings in order to assess the end state of cells. Moreover, we combined immunolabelling information at the last time-point with the time-lapse recording by tracking the immunolabelled cells back through the lineage tree (Fig 4a), using LSTree for further visualization and analysis.

For fixation we fixed the sample at the end of the recording with 4\% PFA, before continuing with the immunolabelling protocol (see Methods Section). Here the pre-patterning of the FEP foil holding the sample was crucial, as without it the Matrigel drops could be easily washed out. To best account for possible organoid drifts, we imaged the entire fixation procedure, so that we could keep track of the organoids during fixation, leading to a recovery after fixation success rate of more than $80 \%$.

On the tissue level, to register the fixed organoids back to their shape at the last time-point of the live recording we used similarity transformations implemented in ITK and available via Elastix $^{20,21}$ (used as a stand-alone tool. For more information, please refer to the Methods Section). As a first example we imaged H2B-mCherry, mem9-GFP organoids until day 3 (Supplementary Movie 3 left). It has been shown that between around day 1.5 intestinal organoids break symmetry through the appearance of the first differentiated cells of the secretory lineage (Paneth cells, Lysozyme). Preceding the appearance of Paneth cells there is the local establishment of a Notch-Delta lateral inhibition event, with future Paneth cells being typically Delta Like Ligand 1 positive $(\operatorname{DLL} 1+)^{6}$. To analyze symmetry breaking, we fixed the organoids at 56 hours and stained for DLL1-Alexa488 and Lys-Alexa647. A cyst was registered to its shape before fixation (as exemplified in Fig $\mathbf{4 b}$ ) and two DLL1+ cells were found (one of them also Lys+), which were followed back over the entire lineage tree while observing their relative position on the segmentation meshes (Fig 4c-e, Supplementary Movie 3 right). Intriguingly, the two DLL1+ cells are two sister cells that were formed at the end of the division from generation 5 to generation 6 , around 10 hours before fixation.

Since both cells show DLL1 but only one is Lys+, one hypothesis was that before generation 5 the cell already starts differentiating towards the secretory lineage (DL11+), but after division one of the daughter cells is differentiating to Paneth cell $(\mathrm{Lyz}+)$. To follow cellular dynamics and changes of features of these specific cells in their spatial environment we analyzed nuclei and cell volumes (extracted with LSTree) per generation of the backtracked cells from generation 5 and 6 and compared them to all of the other cells during the same generations 
262 (Fig 4f). Interestingly, cellular and nuclear volumes of the backtracked cells do not seem to 263 deviate relative to each other during generation 5 . After cell division and entering generation

264 6, however, the nuclei volumes of both DLL1+ sister cells show an increased relative difference to one another, with the Lys+ cell having a slightly larger nucleus.

Next, we evaluated the dynamics of neighbor exchange by cross-checking the closest cells to a backtracked cell(s) of interest at each time-point with LSTree. We examined if the progeny of these two sister cells had high level of mixing with other cells during cyst growth. From the visualization of the tracked neighbors on the lineage tree and segmented meshes (Fig $\mathbf{4 g}$ ), it is apparent that neighbor exchanges, although distributed across the tree, do not happen often nor with many different cells, keeping an average of 5 cells.

The above results show that, by combining our light-sheet framework with standard fixation and registration techniques we can broaden the level of functional information, bridging it to the dynamical processes during live imaging. Consequently, we were capable of dissecting some initial dynamical elements preceding the formation of DLL1+ and Paneth cells in the context of the entire organoid development, analyzing the process of symmetry-breaking events across biological scales.

\section{Nuclei merging events during organoid growth}

From our backtracking example we noticed that one cell undergoes multiple rounds of failed division, with two daughter cells merging before a new division starts (Fig 5a). Upon further inspection of the other 6 lineage trees, we realized that the majority of the datasets contained at least one merging event during early phase of organoid growth whereby two sister nuclei, at the end of their cell cycle, divided again into two instead of into four nuclei. To investigate whether a failed division during the previous mitotic cycle was causing these nuclei merging, we examined the last step of the previous cell division. In all cases there was a problem during late cytokinesis, with the two sister cells never fully separating (Fig $\mathbf{5 a , b}$ ). Nuclear volume for all the daughters arising after the merging event is clearly increased and cell volume followed the same behavior, roughly doubling in tetraploid cells (Fig $\mathbf{5 c , d}$ ). To dismiss the possibility that these mitotic failures are caused by phototoxic effects of the imaging itself, we performed a time-course experiment with wild-type organoids grown from single cells under same medium conditions as the live recordings. We fixed organoids at days 2 and 3 , staining them for e-Cadherin and DAPI to have comparable visual information. Despite the lack of continuous illumination, the resulting data showed many cysts with polynucleated cells, as well as cells with enlarged nuclei (Fig 5e).

Surprisingly, the progeny of a cell that underwent cytokinesis failure can lead to cells that remain part of the epithelium until the end of the recordings, when we can observe fully budded organoids or mature enterocysts (end of day 4) (Fig $\mathbf{5 f}, \mathbf{g})$. Yet, comparing to unaffected parts of the trees, this binucleation progeny typically has higher probability to be extruded into the lumen ( $\sim 6 \%$ for merged progeny against $\sim 5 \%$ for other cells). Another intriguing observation is that the remaining $54 \%$ of the cells are not localized to the crypt but to the villus (Fig $\mathbf{5 f}$, Supplementary Fig $\mathbf{5}$ ). This is an interesting result, as it suggest that there are mechanisms to maintain tissue integrity in the stem cell niche avoiding damaged cells in the crypt. 
307 hypothesis on the role of mitotic failure in regeneration as LATS1 and Yap1 are master 308 regulators of regenerative response of the intestinal epithelium. Analysis of RNAseq from 309 previous studies ${ }^{6}$, show decrease in Lats1 expression during initial days of organoid growth. 310 The first days of organoid growth recapitulate the regenerative response by upregulating 311 Yap1 and downregulating Lats1. Lats1 perturbations and a time-course imaging of Lats1 312 double knockouts ${ }^{6}$ showed several cysts with double nucleated cells that result from mitotic 313 failures (Fig $\mathbf{5 h}$ ). To further perturb the system at another level, we maintained Yap1 314 activation through inhibition of $\mathrm{RXR}^{7}$ and we observed consistent problems during mitosis, 315 including cytokinetic failures (Fig 5i), where daughter nuclei come together after being fully 316 separated (Supplementary Movie 4).

317 Taken together, through the multi scale approach of 3D segmentation, feature extraction and 318 lineage tree analysis we were able to identify consistent polyploidy events during early intestinal organoid development and the fate of their progeny. Our framework allowed us to bridge the observed mitotic defects across scales towards the tissue scale, showing the end fate of the merged cells progeny and spatially locating them onto the mature organoid morphology. This is shedding new light on the robustness of a regenerative YAP cellular state, questioning the role of polyploidy in intestinal regeneration.

\section{Discussion}

327 Here we have presented a unified light-sheet imaging framework tailored to intestinal organoid development. Named LSTree, it is a comprehensive and light-weight approach that uniquely combines image pre-processing, single cell tracking in the form of entire lineage trees and multiscale segmentation and feature extraction along with a dedicated unified visualization and analysis tool. The framework encompasses pre- and post-processing tools for cropping/registration, denoising and deconvolution with combined on the fly compression, generating high-quality datasets with minimal storage costs. We show that this framework is capable of fully segmenting and tracking intestinal organoids as they grow from single cells for several days, bridging biological scales to the point when the organoid has hundreds of cells. Information on organoid, lumen, cell and nuclei volumes along with other multivariate features can be simultaneously visualized with the lineage tree data and further analyzed through a web-based Digital Organoid Viewer, facilitating a more global understanding of the dynamics acquired at subcellular resolution. The use of specialized neural networks dedicated to multiscale segmentation and lineage tree predictions allow the framework to be plastic enough to handle different kinds of live-imaging data as well as continuously improve through the utilization of dedicated tools for retraining of the models. Furthermore, the training architectures keep the number of parameters and computational resources to a minimum, relieving the needs for any highly specialized IT infra-structure, as they can be directly used on off-the-shelf workstations.

346 We pushed the usability of our framework a step further by combining it with standard fixation techniques after live imaging via the development of sample holder patterned with a cold stamp technique, allowing the tracking of immunolabelled cells back in time and space and compare their features to all of the other cells over the entire organoid growth. Unlike 
lineage tracing in single cell RNA sequencing, which clusters cells only by their RNA phenotypic fingerprint or a barcode ${ }^{25}$, with our approach we can focus on the missing spatiotemporal organization in a causal way, as we follow the same cells over time. Consequently, we can now address, in unprecedented detail, the evolution of cells that can give rise to the first symmetry breaking event, keeping track of local interaction within the whole organoid.

Last but not least, we present for the first time the appearance of cells having cytokinesisrelated mitotic errors leading to binucleation during early organoid growth. With LSTree we were able of rapidly verifying these errors across a multitude of different long-term recordings, showing that they are a consistent feature during early cyst growth. Interestingly, polyploidy has been associated with wound healing after injury ${ }^{26}$. In the liver, polyploid cells seem to have a tumor-supressor role with polyploid cells occurring mostly due to cytokinesis failure and endoreduplication ${ }^{27,28}$. However, polyploid hepatic cells are mostly quiescent and do not divide unless the liver undergoes regenerative process due to a lesion. In contrast, our observed bi-nucleated cells do not undergo cell cycle arrest, but continue to divide for even multiple cycles, either with or without repeated clear mitotic failure. We also show that although these cells may appear in the crypt region during crypt formation (during days 3-4), they typically do not manage to reside in the crypt, whereas cells in the villus region remain part of the epithelium. Similar mitotic errors are also shown with inducible Lats-DKO intestinal organoid lines, as well as in the condition of YAP1 overexpression through inhibition of RXR. Since Lats has a direct implication during cytokinesis, we propose that during organoid growth and intestinal regeneration there must be a balance between high-proliferative regenerative state - which is more error prone - and a counteracting checkpoint at the tissue scale to avoid mutations in the stem cell compartment. This way tissue integrity can be achieved fast, with any remaining mutations in the villus being eventually shed off via e.g. anoikis when cells reenter homeostasis.

In conclusion, with LSTree we can cross biological scales with unprecedented detail, as we can follow particular subcellular behaviors while keeping track on the entire tissue development over long periods of time. The usability of the tools presented can go far beyond the examples shown here, as they can be compatible with different light-sheet modalities making this framework also very useful in the study of other 3D cell cultures. In fact, we believe that the LSTree is a first step towards a comprehensive and quantitative framework dedicated to the creation of fully digital organoid maps, so that the intrinsic culture variabilities can be overcome with the creation of averaged organoids to be used as landmarks for future studies.

\section{Organoid lines}

All animal experiments were approved by the Basel Cantonal Veterinary Authorities and conducted in accordance with the Guide for Care and Use of Laboratory Animals. Male and female outbred mice between 7 and 15 weeks old were used for all experiments. Mouse lines used: C57BL/ 6 wild type (Charles River Laboratories), H2B-mCherry C57BL/6 x C3H F1 females heterozygous for H2B-mCherry (kind gift from T. Hiragi lab, EMBL), Lats $1 \Delta / \Delta$; Lats $2 \Delta / \Delta$ (LATS 
Canada) ${ }^{29}$. For H2b-mCherry/mem9-GFP organoids, H2b-mCherry organoids were infected with LV.EF1.AcGFP1-Mem-9 lentivirus particle (Clontech, Takara Bio USA).

\section{Organoid culture}

396 Organoids were generated from isolated crypts of the murine small intestine as previously described $^{5}$. In brief, the section of the initial part of the small intestine was opened lengthwise, cleaned with cold PBS and, after removal of villi by scraping with a cold glass slide, sliced into small fragments roughly $2 \mathrm{~mm}$ in length. The tissue was then incubated in $2.5 \mathrm{mM}$ EDTA/PBS at $4{ }^{\circ} \mathrm{C}$ for 30 min with shaking. Supernatant was removed and pieces of intestine were re-suspended in DMEM/F12 with 0.1\% BSA. The tissue was then shaken vigorously. To collect the first fraction, the suspension was passed through a $70 \mu \mathrm{m}$ strainer.

The remaining tissue pieces were collected from the strainer and fresh DMEM/F12 with $0.1 \%$ BSA was added, followed by vigorous shaking. The crypt fraction was again collected by passing through a $70 \mu \mathrm{m}$ strainer. In total, 4 fractions were collected. Each fraction was centrifuged at $300 \mathrm{~g}$ for $5 \mathrm{~min}$ at $4{ }^{\circ} \mathrm{C}$. Supernatant was removed and the pellet was resuspended into Matrigel with medium (1:1 ratio) and plated into 24 well plates. Organoids were kept in IntestiCult Organoid Growth Medium (STEMCELL Technologies) with $100 \mu \mathrm{g} / \mathrm{ml}$ Penicillin-Streptomycin for amplification and maintenance.

\section{Time course experiments of fixed organoid samples}

412 Organoids were collected 5-7 days after passaging and digested with TrypLE (Thermo Fisher Scientific) for $20 \mathrm{~min}$ at $37{ }^{\circ} \mathrm{C}$. Dissociated cells were passed through a cell strainer with a pore size of $30 \mu \mathrm{m}$ (Sysmex). For all experiments, single alive cells were sorted by FACS (Becton Dickinson FACSAria cell sort or Becton Dickinson Influx cell sorter). Forward scatter and side scatter properties were used to remove cell doublets and dead cells. Sorted cells were collected in ENR medium composed of advanced DMEM/F-12 with 15mM HEPES (STEM CELL Technologies) supplemented with $100 \mu \mathrm{g} / \mathrm{ml}$ Penicillin-Streptomycin, 1×Glutamax (Thermo Fisher Scientific), 1×B27 (Thermo Fisher Scientific), 1xN2 (Thermo Fisher Scientific), 1mM N-acetylcysteine (Sigma), $500 \mathrm{ng} / \mathrm{ml}$ R-Spondin (kind gift from Novartis), $100 \mathrm{ng} / \mathrm{ml}$ Noggin (PeproTech) and $100 \mathrm{ng} / \mathrm{ml}$ murine EGF (R\&D Systems). Collected cells were mixed with Matrigel (Corning) in a medium to Matrigel ratio of 1:1. In each well of a 96 well plate, $3 \mu \mathrm{l}$ droplets with ca. 3000 cells were seeded. After 20 min of solidification at $37^{\circ} \mathrm{C}, 100 \mu \mathrm{l}$ of medium was overlaid. From day 0 to day 1 , ENR was supplemented with $20 \%$ Wnt3a-conditioned medium (Wnt3a-CM), $10 \mu \mathrm{M}$ Y-27632 (ROCK inhibitor, STEMCELL Technologies) and $3 \mu \mathrm{M}$ of CHIR99021 (GSK3B inhibitor, STEMCELL Technologies, cat \# 72054). From day 1 to 3 ENR was supplemented with 20\% Wnt3a-CM and 10 $\mu \mathrm{M} Y$-27632. From day 3 to 5, only ENR was added to the cells. Wnt3a-CM was produced in-house by Wnt3a L-cells (kind gift from Novartis).

\section{Fixed sample preparation and time-course imaging}

Organoids are embedded in a Matrigel droplet. Due to the nature of the droplet, individual organoids are located at different heights in the Matrigel drop. To allow imaging of all organoids within a similar z-range, each 96-well plate was centrifuged at $3000 \mathrm{rpm}$ for $10 \mathrm{~min}$ 
experiments, organoids were permeabilized with 0.5\% Triton X-100 (Sigma-Aldrich) for $1 \mathrm{~h}$ and blocked with 3\% Donkey Serum (Sigma-Aldrich) in PBS with 0.1\% Triton X-100 for $1 \mathrm{~h}$. For the images in Figure 5e, membrane staining with E-Cadherin (BD Biosciences, \# 610182) was done at 1:300 ratio in Blocking buffer for 20 hours at $4^{\circ} \mathrm{C}$. DAPI staining was performed at concentration of $300 \mathrm{nM}$ for $30 \mathrm{~min}$ at room temperature. All secondary antibodies were added at 1:300 for 1 hour in room temperature. Cell nuclei were stained with $20 \mu \mathrm{g} / \mathrm{ml}$ DAPI (4',6-Diamidino-2- Phenylindole, Invitrogen) in PBS for 15 min. High-throughput imaging was done with an automated spinning disk microscope from Yokogawa (CellVoyager 7000S), with an enhanced CSU-W1 spinning disk (Microlens-enhanced dual Nipkow disk confocal scanner), a 40x (NA = 0.95) Olympus objective, and a Neo sCMOS camera (Andor, 2,560 $\times 2,160$ pixels). For imaging, an intelligent imaging approach was used in the Yokogawa CV7000 (Search First module of Wako software). For each well, one field was acquired with $2 x$ resolution in order to cover the complete well. This overview fields were then used to segment individual organoids on the fly with a custom written ImageJ macro which outputs coordinates of individual organoid positions. These coordinated were then subsequently imaged with high resolution $(40 x, N A=0.95)$. For each site, $z$-planes spanning a range up to $140 \mu \mathrm{m}$ were acquired. For the data in Figure 5e $2 \mu \mathrm{m}$ z-steps were used.

\section{Inhibition experiments}

\section{Lats-DKO}

Analysed data stems from a previous publication ${ }^{6}$, with Lats DKO organoids dissociated into single cells and plated into 96 well plates, fixed and stained with DAPI following the published protocols. Tamoxifen induction (1:1000) was kept in the medium until fixation time.

\section{RXRi}

RXR inhibition was achieved by adding the Cpd2170 RXR antagonist ${ }^{7}$ compound at 1:2000 ratio to the medium from the moment single cells were seeded onto the light-sheet holder. The compound was kept throughout the data acquisition. Organoids used for this experiment had been infected with H2B-iRFP670 for live nuclei labeling.

\section{Light-sheet recordings}

\section{6}

\section{Sample preparation}

H2b-mCherry / mem9-GFP and H2B-iRFP670 organoids were collected and digested with TrypLE (Thermo Fisher Scientific) for $20 \mathrm{~min}$ at $37^{\circ} \mathrm{C}$. Alive double positive (mCherry/GFP) cells were sorted by FACS and collected in medium containing advanced DMEM/F-12 with 15 mM HEPES (STEM CELL Technologies) supplemented with $100 \mu \mathrm{g} / \mathrm{ml}$ Penicillin-Streptomycin, 1×Glutamax (Thermo Fisher Scientific), 1×B27 (Thermo Fisher Scientific), 1xN2 (Thermo Fisher Scientific), $1 \mathrm{mM} \mathrm{N}$-acetylcysteine (Sigma), 500ng/ml R-Spondin (kind gift from Novartis), 100 $\mathrm{ng} / \mathrm{ml}$ Noggin (PeproTech) and $100 \mathrm{ng} / \mathrm{ml}$ murine EGF (R\&D Systems). 2500 cells were then embedded in 5 ul drop of Matrigel/medium in 60/40 ratio. Drops were placed in the imaging chamber and incubated for 20 min before being covered with $1 \mathrm{ml}$ of medium. For the first three days, medium was supplemented with $20 \%$ Wnt3a-CM and $10 \mu \mathrm{M}$ Y-27632 (ROCK inhibitor, STEMCELL Technologies). For the first day, in addition, $3 \mu \mathrm{M}$ of CHIR99021 
(STEMCELL Technologies) were supplemented. After 2 hours incubation in a cell culture incubator the imaging chamber was transferred to the microscope kept at $37 \mathrm{C}$ and $5 \% \mathrm{CO} 2$.

For all light-sheet experiments a LS1-Live dual illumination and inverted detection microscope from Viventis Microscopy Sàrl was used. Different single cells were selected as starting positions and imaged every $10 \mathrm{~min}$ for up to 5 days. A volume of $150-200 \mu \mathrm{m}$ was acquired with a Z spacing of $2 \mu \mathrm{m}$ between slices and $100 \mathrm{~ms}$ exposure time for each slice. Laser intensity was kept to a minimum necessary to still obtain reasonable signal to noise from the raw data, while keeping phototoxicity to a minimum possible. Medium was exchanged manually under the microscopy every day.

\section{Fixation on time-lapse recordings}

Organoids are embedded in $5 \mu \mathrm{m}$ Matrigel droplets which are deposited at equal distances on top of the FEP foil of the light-sheet sample holder. After live imaging is done, the medium is replaced by $4 \%$ PFA in PBS, and left in the chamber for maximum 30 minutes at $37^{\circ} \mathrm{C}$ in the microscope. After fixation the organoids were permeabilized with 0.5\% Triton X-100 (SigmaAldrich) for $1 \mathrm{~h}$ and blocked with 3\% Donkey Serum (Sigma-Aldrich) in PBS with 0.1\% Triton X-100 for $1 \mathrm{~h}$. For the images in Figure 4, the cyst was stained with DLL1 antibody (R\&D Systems, \# AF3970) at 1:100 ratio and left overnight at $4^{\circ} \mathrm{C}$. For Lysozyme (Dako, \# A0099) we used a 1:400 ratio for 3 hours at room temperature.

499

\section{Registration for back-tracking after fixation of time-lapses}

501

Since PFA fixation causes the Matrigel droplet to flatten, we perform imaging while fixation

502 is taking place. Typically we observe no change within the first 5 minutes, whereas after that there is a sudden increase in organoid movement towards the bottom of the sample holder. To take this into account, we increased the imaging volume and step size to be able to encompass a larger volume and still track the organoid. For the data in Figure 4 we increased stack size from $150 \mu \mathrm{m}$ and $2 \mu \mathrm{m}$ step size to $300 \mu \mathrm{m}$ at $3 \mu \mathrm{m}$ step size. However, larger values can also be used.

508 Nonetheless, the flattening of the droplet will lead the organoids to rotate or translate in 509 space. Furthermore, PFA fixation also changes the shape of tissue samples by shrinking or swelling. To bridge the translational, rotational and rescaling of the organoids during fixation procedures, we registered fixed organoids using Elastix v4.900 (https://elastix.lumc.nl/). Since Elastix can be directly installed from the repository as pre-compiled libraries, we refrained from embedding the registration into LSTree, and left it as a stand-alone tool. For all registrations using the similarity transform, a base parameter file set for performing similarity transformations was used and eventually modified so that best results could be achieved.

518 Pre-processing steps rely mostly on cropping and registration, denoising, and deconvolution 519 steps. Although not part of LSTree itself, improvements in the microscope software (on-the- 
520 fly LZW compression, position dependent illumination alignment) were performed in 521 collaboration with Viventis Microscopy Sàrl and are now part of their current microscope 522 software.

523 Detailed information regarding pre-processing, segmentation strategies and feature 524 extraction can be found in Supplementary Text.

525

526

527 


\section{Acknowledgements}

529 We thank M. Rempfler for helpful discussions on framework implementation and support, D.

530 Vischi, E. Tagliavini and Sjoerd van Eeden for IT support, Q. Yang for discussions on pre-

531 processing, S. Xie for helpful discussions on biological aspects, A. Peters for light-sheet, I.

532 Lukonin for help with RXR compound, H. Kohler for sorting, P. Argast for help with building

533 the FEP foil aluminum stamp, C. Tsiairis, L. Gelman and laboratory members for reading the

534 manuscript. Funding: EMBO (ALTF 571-2018 to G.M.), SNSF (POOP3_157531 to P.L.). This

535 work received funding from the ERC under the European Union's Horizon 2020 research and

536 innovation programme (grant agreement no. 758617).

\section{Author information}

539 Author notes

540 These authors contributed equally: Gustavo de Medeiros, Raphael Ortiz

541

542

Affiliations

543 Friedrich Miescher Institute for Biomedical Research (FMI), Basel, Switzerland

544 Gustavo de Medeiros, Raphael Ortiz, Francisca Maurer \& Prisca Liberali

545 University of Basel, Basel, Switzerland

546 Prisca Liberali

547 Viventis Microscopy Sàrl, EPFL Innovation Park, Lausanne, Switzerland

548 Andrea Boni, Petr Strnad

549

550

\section{Contributions}

551 P.L. and G.M. conceived and P.L. supervised the study, P.L., G.M., and A.B. designed the 552 experiments, F.M., G.M. cultured the organoids, G.M. and A.B. recorded the time-lapses, P.S. 553 wrote and implemented compression and position dependent illumination code into 554 microscope software, A.B. created first Mastodon trees, R.O. wrote LSTree framework with 555 the support of G.M., G.M. performed time-course experiment. G.M., R.O. and P.L. analysed 556 the data, G.M., R.O. and P.L. wrote the paper.

558 Corresponding author

559 Correspondence to Prisca Liberali. 
561

562

563

564

565

566

567

5681

569

570

571

572

573

$574 \quad 3$

575

576

577

578

$579 \quad 5$

580

581

582

$583 \quad 7$

584

585

586

587

588

589

590

591

592

593

594

595

596

\section{Ethics declarations}

\section{Competing interests}

A.B. and P.S. are co-founders of Viventis Microscopy Sàrl that commercializes the light-sheet microscope used in this study.

\section{References}

1 Mayr, U., Serra, D. \& Liberali, P. Exploring single cells in space and time during tissue development, homeostasis and regeneration. Development 146, dev176727, doi:10.1242/dev.176727 (2019).

2 Zinner, M., Lukonin, I. \& Liberali, P. Design principles of tissue organisation: How single cells coordinate across scales. Current Opinion in Cell Biology 67, 37-45, doi:10.1016/j.ceb.2020.07.004 (2020).

Xavier Da Silveira Dos Santos, A. \& Liberali, P. From single cells to tissue selforganization. The FEBS Journal 286, 1495-1513, doi:10.1111/febs.14694 (2019).

4 Bankaitis, E. D., Ha, A., Kuo, C. J. \& Magness, S. T. Reserve Stem Cells in Intestinal Homeostasis and Injury. Gastroenterology 155, 1348-1361, doi:10.1053/j.gastro.2018.08.016 (2018).

Sato, T. et al. Single Lgr5 stem cells build crypt-villus structures in vitro without a mesenchymal niche. Nature 459, 262-265, doi:10.1038/nature07935 (2009).

6 Serra, D. et al. Self-organization and symmetry breaking in intestinal organoid development. Nature 569, 66-72, doi:10.1038/s41586-019-1146-y (2019).

7 Lukonin, I. et al. Phenotypic landscape of intestinal organoid regeneration. Nature 586, 275-280, doi:10.1038/s41586-020-2776-9 (2020).

8 Yang, Q. et al. Cell fate coordinates mechano-osmotic forces in intestinal crypt morphogenesis. bioRxiv pre-print server, doi:10.1101/2020.05.13.094359 (2020).

9 Alladin, A. et al. Tracking cells in epithelial acini by light sheet microscopy reveals proximity effects in breast cancer initiation. elife 9, doi:10.7554/elife.54066 (2020).

10 Kok, R. N. U. et al. OrganoidTracker: Efficient cell tracking using machine learning and manual error correction. PLOS ONE 15, e0240802, doi:10.1371/journal.pone.0240802 (2020).

11 Hof, L. et al. Long-term live imaging and multiscale analysis identify heterogeneity and core principles of epithelial organoid morphogenesis. BMC Biology 19, doi:10.1186/s12915-021-00958-w (2021).

12 Dumortier, J. G. et al. Hydraulic fracturing and active coarsening position the lumen of the mouse blastocyst. Science 365, 465-468, doi:10.1126/science.aaw7709 (2019). 
59713 Rossi, G. et al. Capturing Cardiogenesis in Gastruloids. Cell Stem Cell 28, 230-240.e236, doi:10.1016/j.stem.2020.10.013 (2021).

14 Krull, A., Buchholz, T.-O. \& Jug, F. Noise2Void - Learning Denoising from Single Noisy

600 Images. arXiv pre-print server, doi:arxiv:1811.10980 (2019). toolkit for high dimensional fluorescent microscopy imaging. BMC Bioinformatics 20, doi:10.1186/s12859-019-3055-3 (2019).

604 (Springer International Publishing, 2020).

606

Ulman, V. et al. An objective comparison of cell-tracking algorithms. Nature Methods

607

608 14, 1141-1152, doi:10.1038/nmeth.4473 (2017).

609

18 Moen, E. et al. Accurate cell tracking and lineage construction in live-cell imaging experiments with deep learning (Cold Spring Harbor Laboratory, 2019).

611

19 Sugawara, K., Cevrim, C. \& Averof, M. Tracking cell lineages in 3D by incremental deep learning (Cold Spring Harbor Laboratory, 2021).

613

Klein, S., Staring, M., Murphy, K., Viergever, M. A. \& Pluim, J. P. W. elastix: A Toolbox

614 for Intensity-Based Medical Image Registration. IEEE Transactions on Medical Imaging 29, 196-205, doi:10.1109/TMI.2009.2035616 (2010).

21 Shamonin, D. Fast parallel image registration on CPU and GPU for diagnostic classification of Alzheimer's disease. Frontiers in Neuroinformatics 7, doi:10.3389/fninf.2013.00050 (2013).

619

Yabuta, N. et al. N-terminal truncation of Lats1 causes abnormal cell growth control

620 and chromosomal instability. Journal of Cell Science 126, 508-520, doi:10.1242/jcs.113431 (2013).

Yang, X. et al. LATS1 tumour suppressor affects cytokinesis by inhibiting LIMK1. Nature

622

623 Cell Biology 6, 609-617, doi:10.1038/ncb1140 (2004).

Lens, S. M. A. \& Medema, R. H. Cytokinesis defects and cancer. Nature Reviews Cancer 19, 32-45, doi:10.1038/s41568-018-0084-6 (2019).

626

25 Wheat, J. C. et al. Single-molecule imaging of transcription dynamics in somatic stem cells. Nature 583, 431-436, doi:10.1038/s41586-020-2432-4 (2020).

628 Losick, Vicki P., Fox, Donald T. \& Spradling, Allan C. Polyploidization and Cell Fusion Contribute to Wound Healing in the Adult $<$ em $>$ Drosophila $</$ em $>$ Epithelium. Current Biology 23, 2224-2232, doi:10.1016/j.cub.2013.09.029 (2013).

630

27 Zhang, S. et al. The Polyploid State Plays a Tumor-Suppressive Role in the Liver.

631 Developmental Cell 44, 447-459.e445, doi:10.1016/j.devcel.2018.01.010 (2018). 3639, doi:10.1242/jcs.016907 (2007). 
bioRxiv preprint doi: https://doi.org/10.1101/2021.05.12.443427; this version posted May 12, 2021. The copyright holder for this preprint

(which was not certified by peer review) is the author/funder, who has granted bioRxiv a license to display the preprint in perpetuity. It is made available under aCC-BY 4.0 International license.

29 Gregorieff, A., Liu, Y., Inanlou, M. R., Khomchuk, Y. \& Wrana, J. L. Yap-dependent

638 

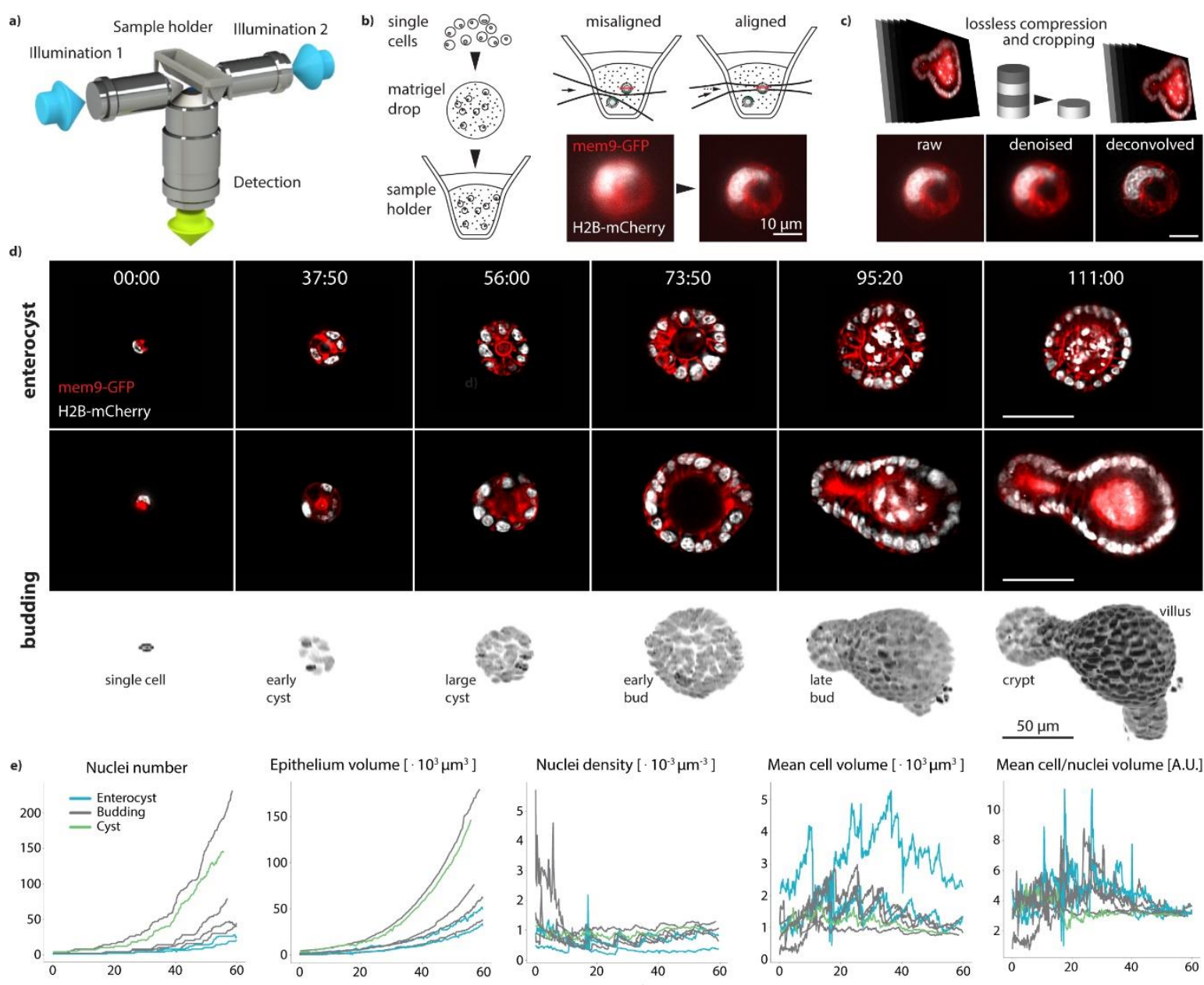

\section{2}

Figure 1: Acquisition of high-resolution 3D organoid images. a) Dual illumination inverted detection light-sheet objective configuration used in all of the recordings. b) Left: sample preparation is performed by mixing single cells dissociated from mature organoids with matrigel and depositing $5 \mathrm{uL}$ drops on the light-sheet sample holder. Right: sample position dependent illumination alignment corrects for possible misalignments of the illumination beam in reaching organoids distributed inside the Matrigel drop, improving SNR. c) Upper row: minimization of storage needs done with on-the-fly compression and further cropping steps. Lower row: denoising and deconvolution steps further improve image quality. d) Timelapse imaging of organoid growth. Top row shows still images of a growing enterocyst, whereas both lower rows show a stereotypical growth of a budding organoid. e) Temporal evolution of nuclei number, epithelium volume, mean cell volume and the ratio between mean cell and mean nuclei volumes for all 7 datasets considered in this work. 

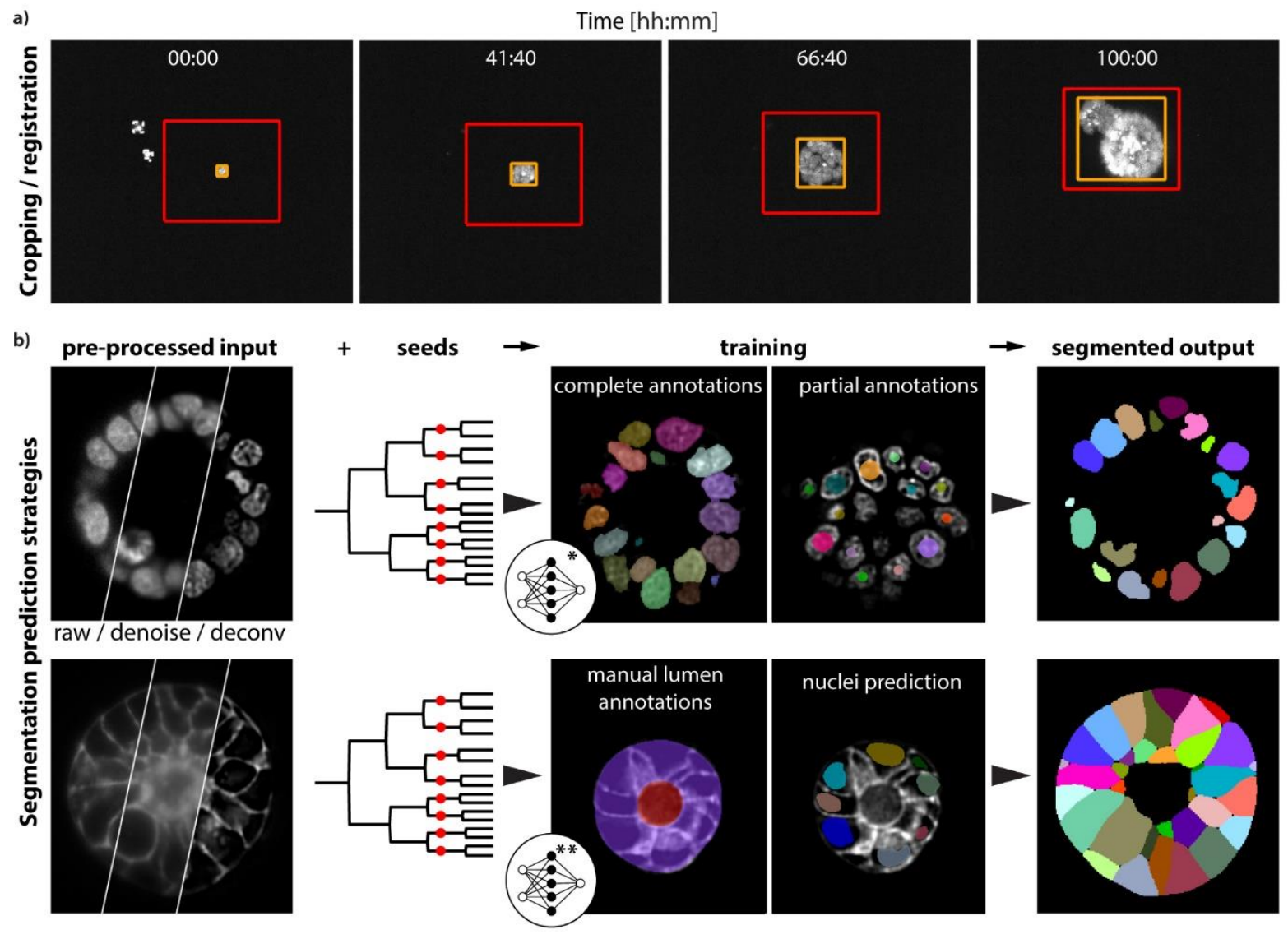

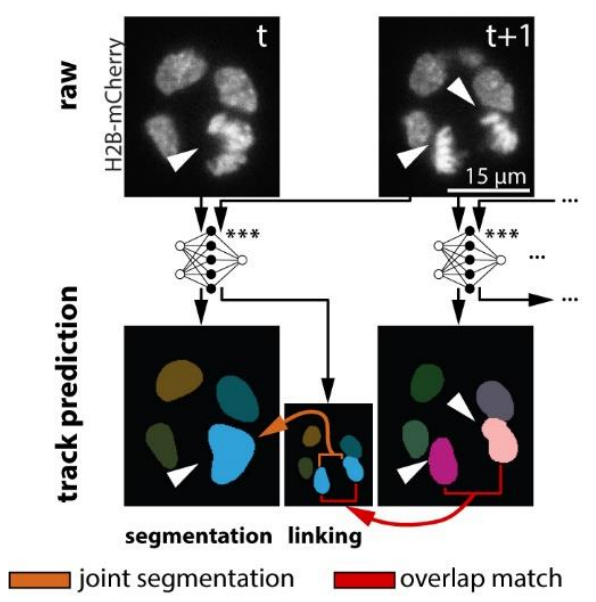

d)

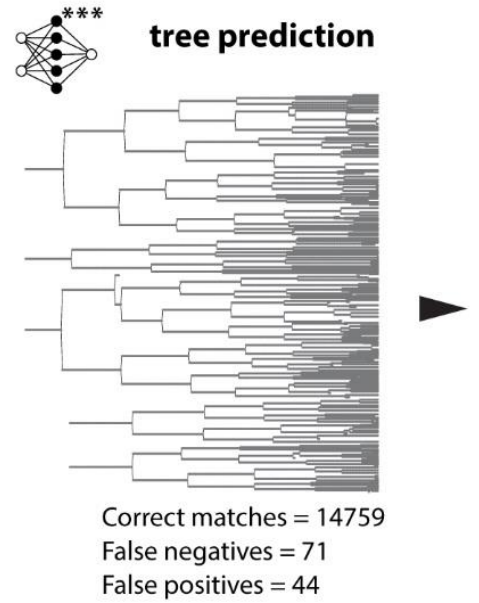

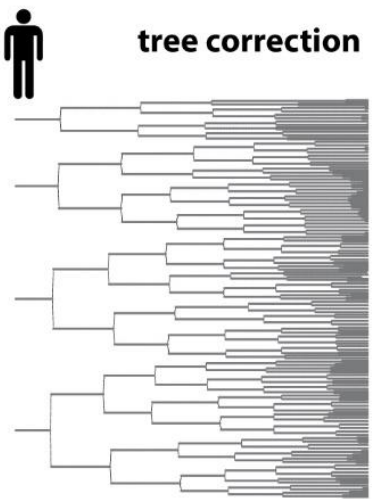

End number of cells $=231$

Figure 2: Cropping, segmentation and tree-prediction strategies underlying LSTree. a) Cropping of datasets is done in a semi-automatic way: selected object of interest is fitted with an orange (best fit for each particular timepoint) and a global red bounding boxes, which can be corrected in 3D. b) Nuclei and cell segmentation strategies. Upper row: denoised and deconvolved input data together with seeds from respective tracking are used as input into the network to predict nuclei volume. The network is trained with both complete and partial annotations. Lower row: Cell volume prediction follows similar input as for nuclei. Main difference is that this second network is trained with supervision of complete manual annotations of lumen and organoid along with the previously done nuclei predictions 
bioRxiv preprint doi: https://doi.org/10.1101/2021.05.12.443427; this version posted May 12, 2021. The copyright holder for this preprint (which was not certified by peer review) is the author/funder, who has granted bioRxiv a license to display the preprint in perpetuity. It is made available under aCC-BY 4.0 International license.

666 themselves. c) Strategy for prediction of lineage trees. Track predictions are done with each 667 consecutive pair of frames. Each pair of frames enter the neural network and produce both 668 the timepoint in question and a linking frame which is used to connect to the next timepoint 669 via overlap match. Linking itself is done via joint segmentation. d) Example of predicted and 670 corrected tree from a budding organoid dataset with the recording starting from two cells. 

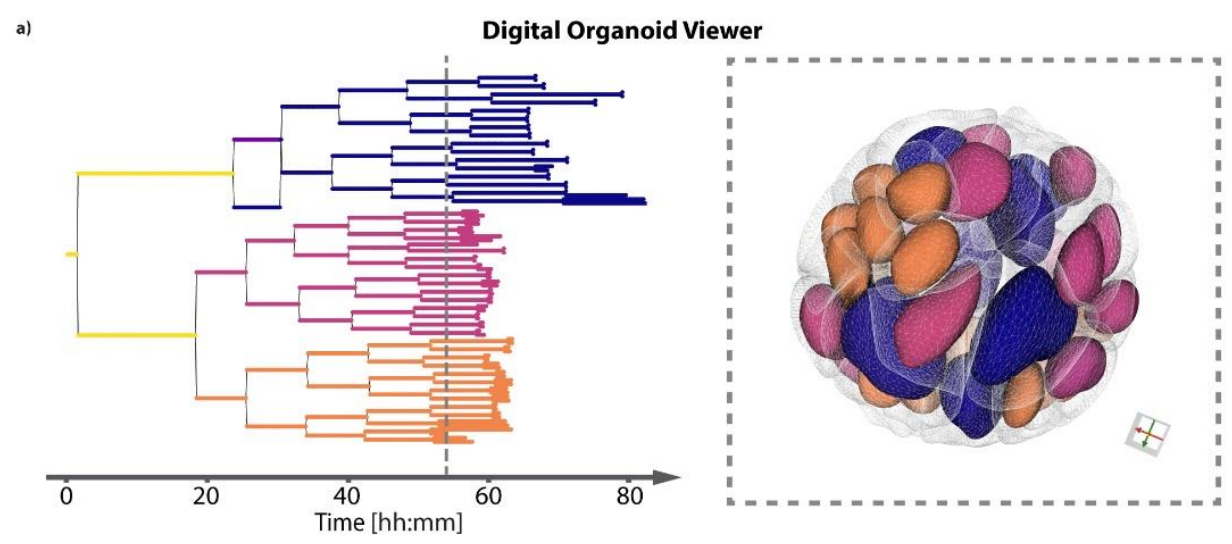

b)

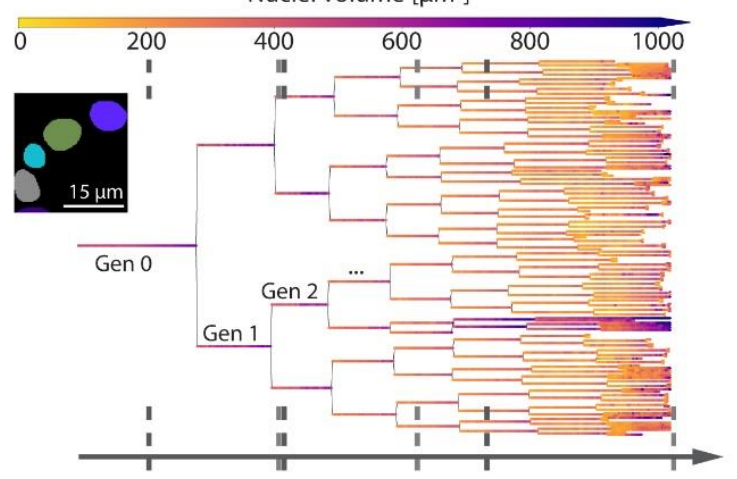

c)

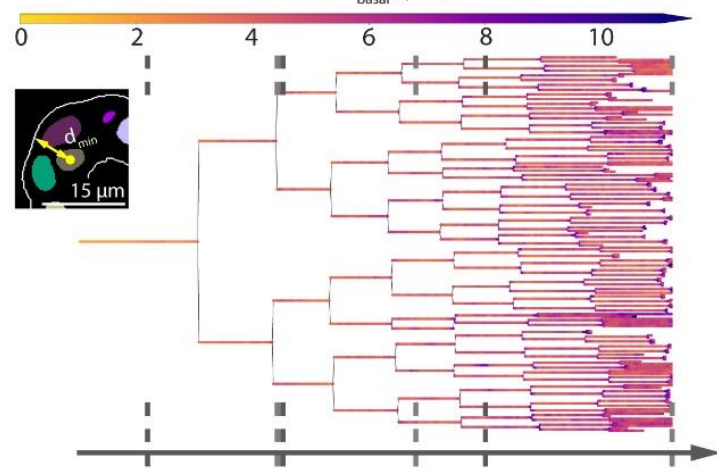

d)

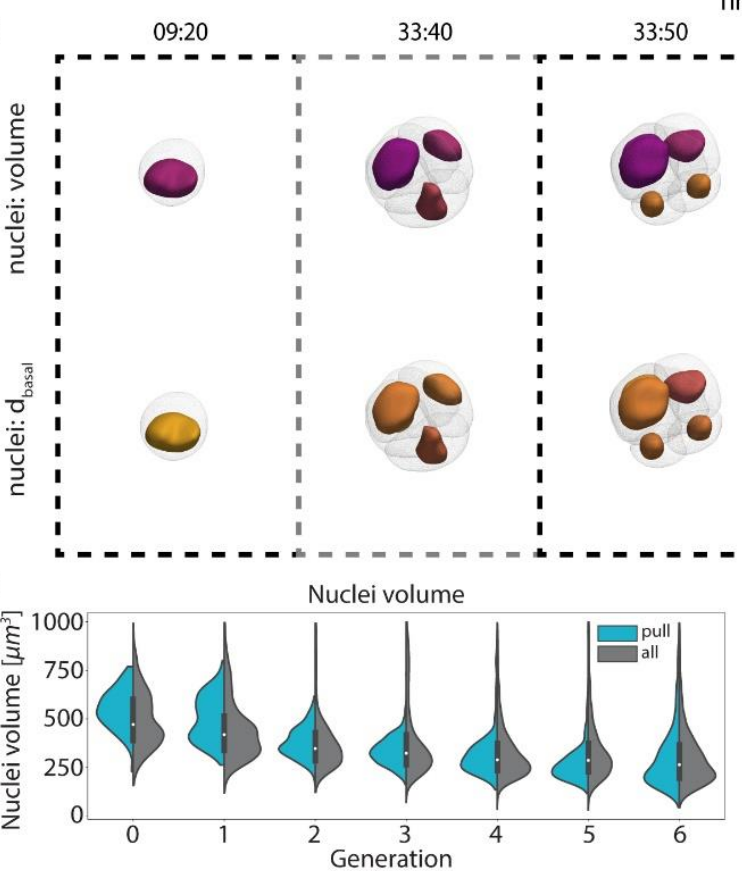

Time [hh:mm]

673 Figure 3: Digital Organoid Viewer. a) Digital organoid viewer is a web-based tool that shows

674 both lineage trees (left) and respective segmented nuclei and cell meshes (right) 675 simultaneously. Color coding of of each data representation can be done in a combined or 676 complementary manner. Here depicted is a color coding based on generation 2, with each of 677 the four cells acquiring different colors being propagated further in time.b) Overlay of the 678 nuclei segmentation as predicted in onto the lineage tree. c) Overlay of distance to basal 
679 membrane onto the lineage tree. d) Visualization of the calculated meshes from nuclei and 680 cell segmentations, overlaying the corresponding values (with corresponding color map) of 681 the features presented in b) and c). The time points chosen are shown via dashed lines on

682 each tree in b) and c). e,f) Extracted values for nuclei volume (e) and for distance to apical 683 membrane (f) of the here exemplified dataset (pull) against all datasets (all) analyzed in this 684 work.

685 

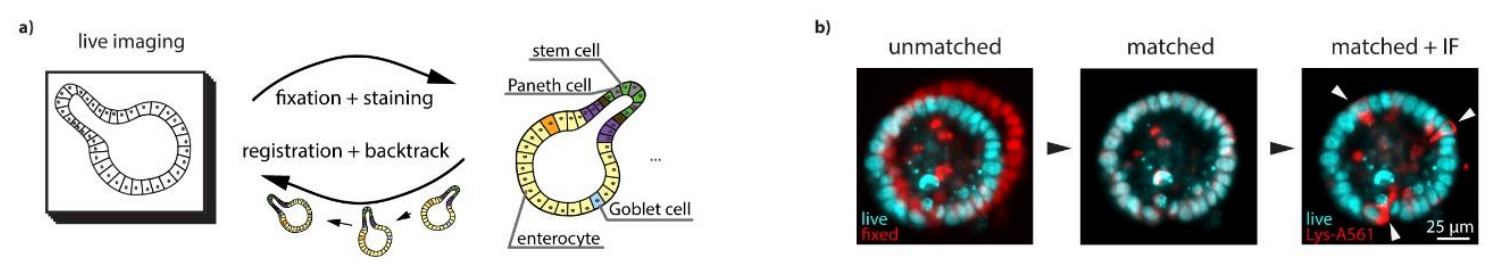

c)
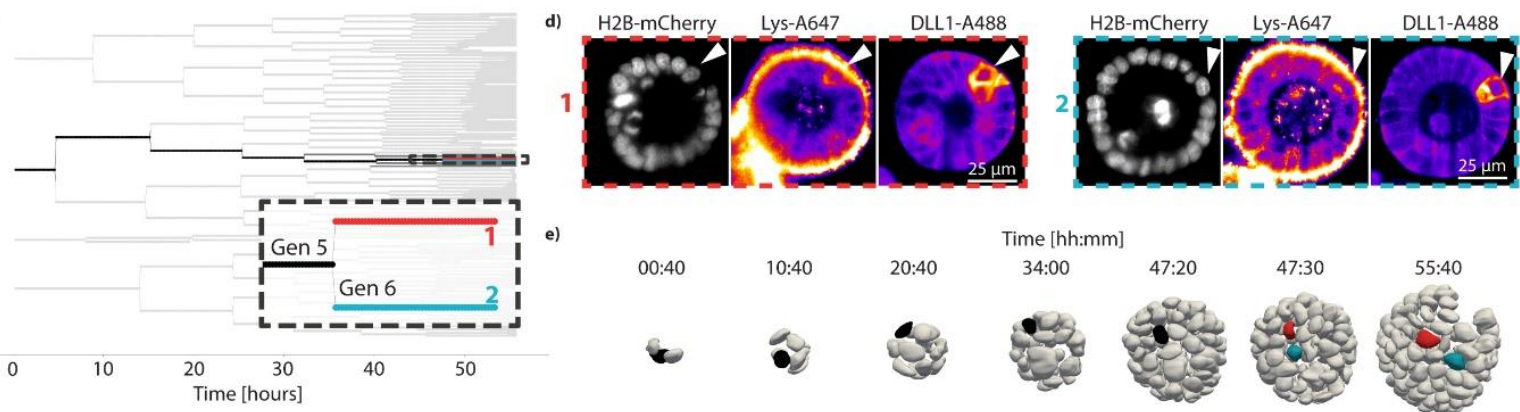

f)

Gen 5

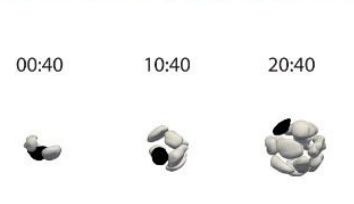

Time [hh:mm]
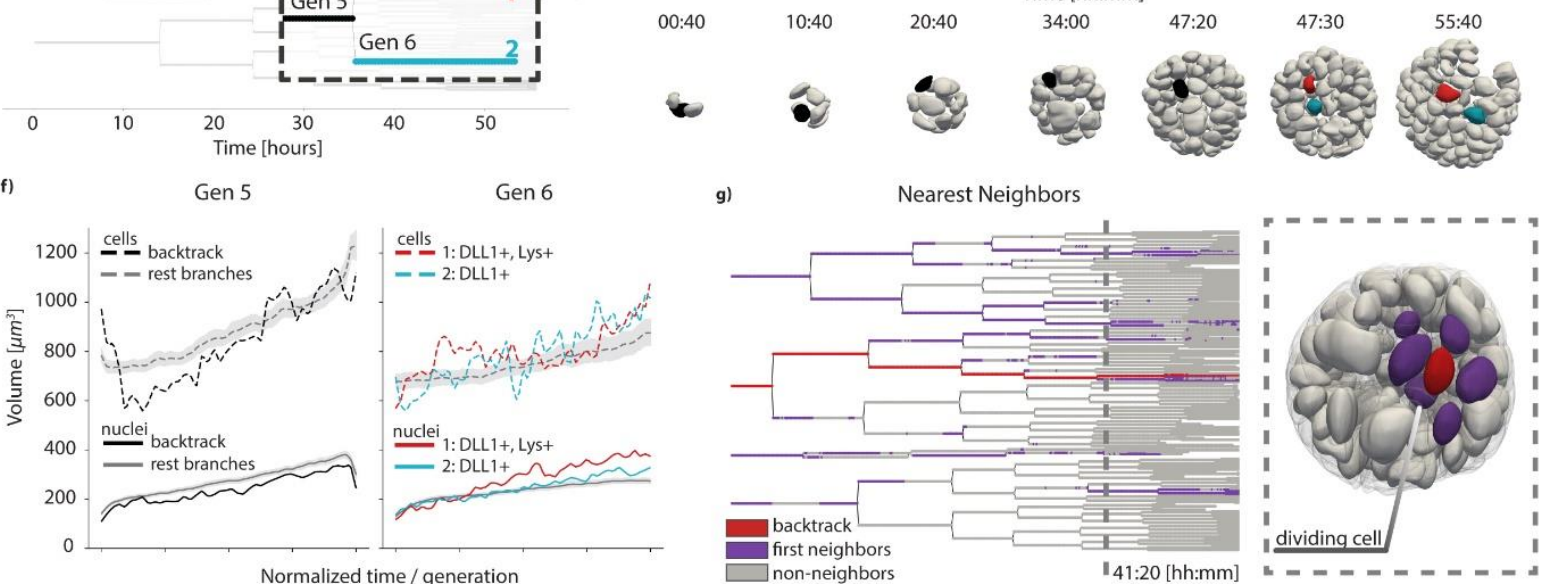

Figure 4: Fixation and backtracking after live-imaging. a) Fixation and backtracking strategy for light-sheet imaging of organoid growth. b) After fixation a registration step is needed in order to overlap the fixed nuclei with the nuclei as shown in the last timepoint of the timelapse. Left: midplane of raw data from last time-point H2B-mCherry recording (cyan) against same plane after fixation (red). Center: registration maps the fixed volume into the last time-point volume. Although overlap is not perfect, it is sufficient to maps each nuclei back. Right: After registration additional information from immunolabelling can be overlayed onto last time-point of the live recording, and so cells of interest can be backtracked. c) Lineage tree depicting the backtracking of two sister cells. d) After fixation, staining for DLL1 and Lysozyme shows two cells expressing these markers. Backtracking of them is shown in a). e) Nuclei volume distribution for the backtracked cells against all other cells per generation. f) Evaluation of nuclei and cell volumes for the backtracked cells against all other cells during generations 5 and 6 , as depicted in c). For all other cells, the mid line corresponds to the mean, whereas the gray region is the standard distribution. g) Nearest neighbor evaluation of the backtracked Lys+ cell (red). All nearest neighbors are depicting in magenta, with all remaining cells in gray. Dashed line on the lineage tree is presented as corresponding segmented meshes on the right. A dividing cell can be recognized by the interkinetic movement of its nucleous further apically. 

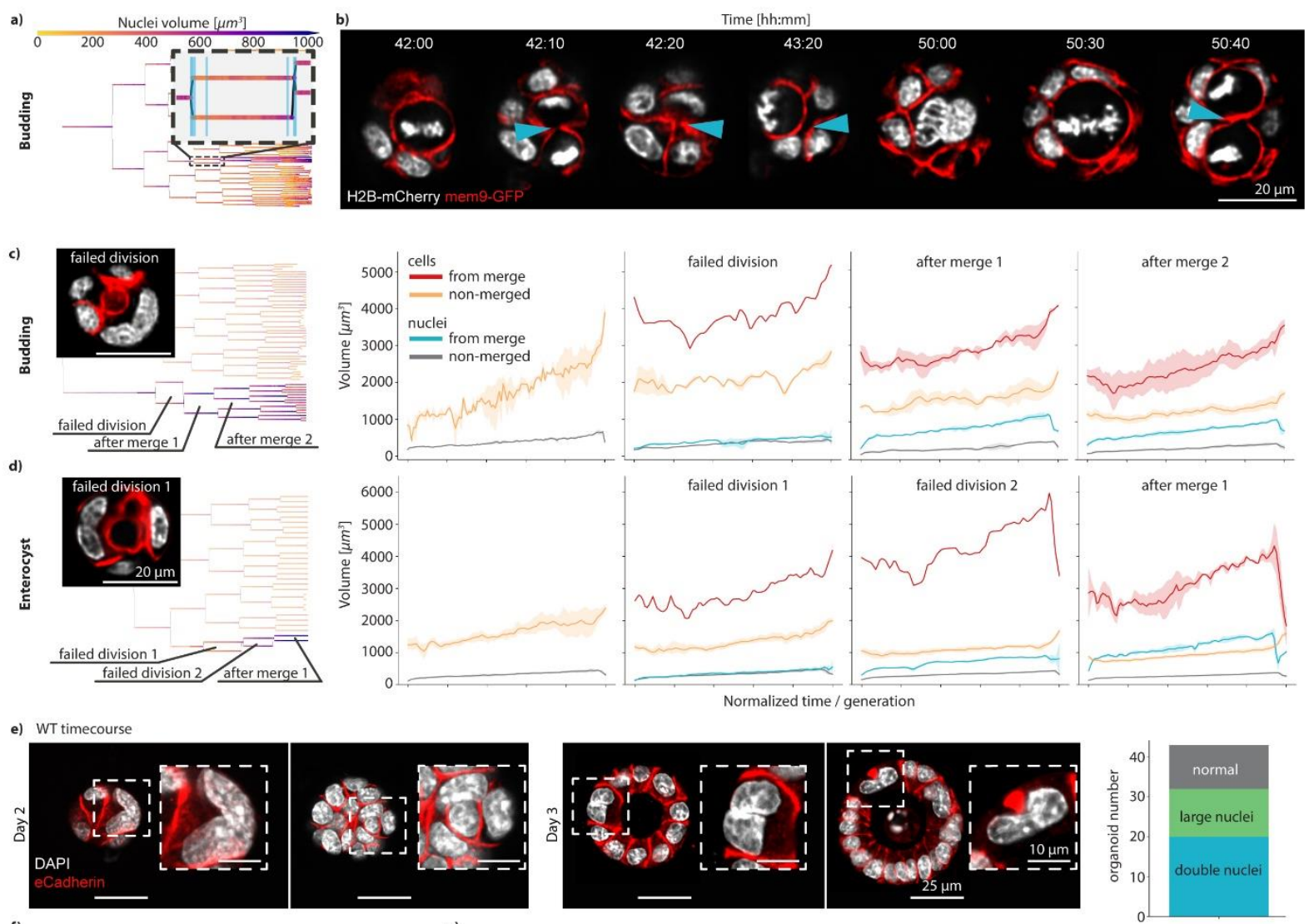

f)

g)
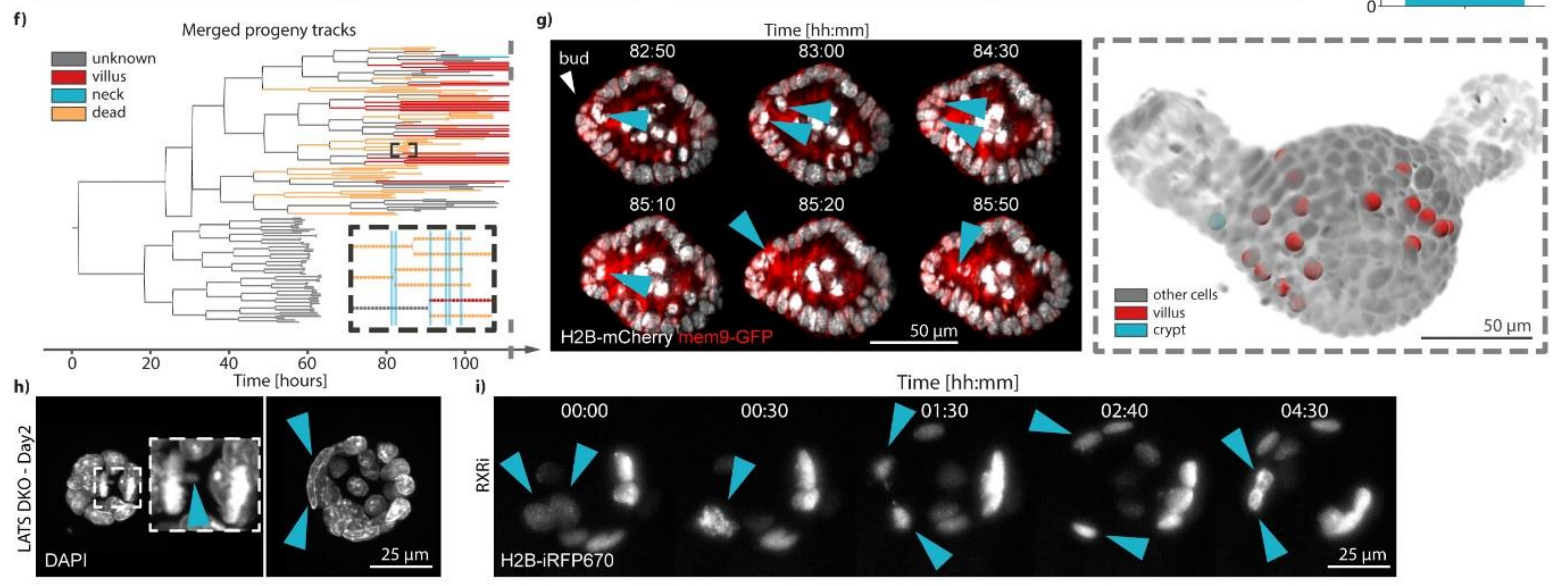

Figure 5: Merging events during early organoid growth. a) Example lineage tree with highlighted insert depicting a cell division where two nuclei divide again into two nuclei. b) Still images of the light-sheet recording related to the dataset in a). Cyan arrows mark the position of a connecting region between the two nuclei until the following division occurs, where cytokinesis is successful. Corresponding locations for each depicted timepoint on the lineage tree are shown as cyan lines in the insert in a). c,d) Examples of different sequence of events following a failed division during budding organoid and enterocyst growth (left), with quantified nuclei and cell volumes for the highlighted events on the trees (right). e) Timecourse data on wild-type intestinal organoids grown from single cells and fixed at days 2 and 3 after seeding. Staining with DAPI and e-Cadherin show binucleated cells and cells with large nuclei, which are quantified on the right.. f) Left: outcomes from merged progeny and cells from other parts of the tree. No cells part of the merged progeny make it to the crypt. 
721 the lumen or basally extruded, unknown corresponds to tracks where the high level of cell

722 packing and/or the low quality of the images made it impossible to continue and know their

723 fate, and alive corresponds to tracks where the cells are still part of the epithelium until the

724 end of the recording. Right: overlay of tracked cells from left panel onto the last time-point

725 of the recording, showing the spatial organization of the cells in the crypt and vilus regions. f)

726 g) Major axis length of all nuclei after a failed division (green) and all other cells (gray). h)

727 Time-course images of Lats1-DKO organoids at day2. Cyan arrows show lagging chromosome

728 during division (left) and binucleated cell (right). i) Still images of an organoid expressing H2B-

729 iRFP670 under RXR inhibitor condition. Cyan arrows follow the moment of cell division of a 730 binucleated cell, with daughter nuclei jumping back to proximity in the end. 